\title{
Existe una relación inversa entre mortalidad y la capacidad funcional medida por Ergometría
}

Exercise capacity and mortality among men referred for exercise testing. Myers J, Prakash M, Froelicher V. N Engl J Med 2002;346:793801

\section{Objetivo}

Evaluar la capacidad funcional como marcador pronóstico de mortalidad en personas sín enfermedad cardiovascular establecida referidas para una Ergometría.

Establecer la influencia de los beta bloqueantes sobre el valor pronóstico de la tolerancia al esfuerzo.

\section{Diseño}

Estudio prospectivo de una cohorte de pacientes referidos para una evaluación ergométrica por motivos clínicos. Seguimiento medio 6.2 años.

\section{Lugar}

Dos centros en Estados Unidos (Universidad de Stanford y el Centro de Veteranos) en Palo Alto, California.

\section{Población}

Se evaluaron 6213 hombres en forma consecutiva

\section{Evaluación de los factores de riesgo}

Se relevaron múltiples variables de la historia clínica como medicación actual, factores de riesgo cardiovascular, examen físico, ECG, edad, BMI, antecedentes pulmonares y antecedentes cardiovasculares, y se midió la capacidad funcional (medida en METs) y otras variables relacionadas a través de una prueba ergométrica en cinta deslizante.

\section{Resultados principales}

De acuerdo con los resultados de la Ergometría y los antecedentes cardiovasculares y pulmonares la población fue dividida en: sujetos normales y sujetos con enfermedad cardiovascular definida. La mortalidad anual promedio fue de $2.6 \%$. Del grupo de los sujetos sanos los sobrevivientes eran de menor edad $(p<0.001)$, tenían un BMI mayor ( $\mathrm{p}: 0.005$ ), su frecuencia cardíaca basal era más baja y durante la Ergometría alcanzaban una FC mayor, una presión arterial sistólica mayor, y la capacidad de ejercicio medida en METs era superior ( $p: 0.001$ ). En el grupo de pacientes con enfermedad cardiovascular definida los hallazgos fueron similares. Ajustadas por edad la variable que mejor predijo muerte de cualquier causa en los sujetos normales fue la capacidad de ejercicio pico, seguida por la cantidad de paquetes año de cigarrillos fumados. Por cada 1 MET de incremento en la capacidad de ejercicio disminuyó un $12 \%$ la mortalidad total. El riesgo relativo de muerte medido entre el quintilo inferior ( $<5 \mathrm{METs}$ de capacidad) y el quintilo superior (capacidad de ejercicio >13 METs en sujetos sín enfermedad cardiaca y $>10$ METs con enfermedad) fué de 4 (IC $95 \% 3$ a 6.8 ) y 4.5 (3.3 a 5.2 IC95\%) respectivamente. Esta diferencia comienza a hacerse significativa a partir de los 8 METs. La capacidad de ejercicio absoluta fue mejor predictora de mortalidad que la capacidad de ejercicio ajustada por edad. No se observó interacción entre los beta-bloqueantes y el poder predictivo de la capacidad de ejercicio.

\section{Conclusiones}

Este trabajo demuestra que la capacidad de ejercicio es un fuerte predictor de mortalidad en sujetos referidos para la realización de una Ergometría por motivos clínicos.

\section{Comentario}

Numerosos estudios epidemiológicos han demostrado la relación inversa entre capacidad de ejercicio y mortalidad en distintas poblaciones asintomáticas. En la década del 50 los estudios de Morris en los trabajadores del transporte público londinense demostró que el nivel de actividad física en el área laboral se relacionaba con la morbi-mortalidad coronaria'. Otros estudios comprobaron lo mismo con respecto a la actividad física durante las horas de ocio. Un estudio retrospectivo de Paffenbarger con los ex alumnos de Harvard demostró que la actividad física actual es la que protege y no la pasada. Posteriormente en el centro de estudios aeróbicos de la Clínica Cooper, Blair y colaboradores demuestran que la modificación del sedentarismo a través de la actividad física normalizaba el riego cardiovascular de dichos sujetos. Una revisión de Powell y col. resumió 54 publicaciones sobre ejercicio y enfermedad coronaria y concluyó lo siguiente: 1 . El riesgo de enfermedad coronaria es en promedio el doble para los sujetos de bajo nivel de actividad física. 2. Parece existir una relación dosis respuesta, a más bajo nivel de actividad física mayor riesgo. 3 . El sedentarismo es un factor de riesgo independiente de los otros factores de riesgo?
No solo los bajos niveles de actividad física se relacionan con aumento de la mortalidad cardiovascular sino de mortalidad global y progresión de numerosas enfermedades crónicas. Si bien el articulo comentado se realizó en hombres, el efecto beneficioso de la actividad física es similar en ambos sexos, como lo demuestran múltiples estudios en mujeres como el estudio de las enfermeras ${ }^{3}$. Lo mismo ocurre con los ancianos, aunque los sujetos mayores de 65 años del estudio eran pocos, otros estudios confirman el beneficio en esta población $n^{4}$. Del análisis del estudio comentado y de lo expuesto en el comentario podemos finalmente concluir que: La Ergometría es una herramienta útil para medir la capacidad funcional de una persona y predecir su riesgo. Siendo el sedentarismo uno de los llamados factores de riego modificables, debemos desde el consultorio aprovechar todas las oportunidades para modificarlo. Nos quedaría por definir el tipo, duración, intensidad y frecuencia de la actividad física a prescribir, variables que deben ser tenidas en cuenta a la hora de cuidar la principal meta para combatir el sedentarismo que es la falta de adherencia ó discontinuidad.

\section{Dr. Diego Iglesias [ Servicio de Cardiología. Hospital Italiano de Buenos Aires ]}

\author{
Bibliografía \\ 1. Morris J, Heady P, Raffle C. Coronary Heart disease and physical activity of work. Lancet 1953;2:1053-1057 \\ 2. Powell K, Thompson P, Caspersen C. Physical activity and the incidence of coronary heart disease. Ann Rev Public Health.1987;8:253-287 \\ 3. Rockhill B, Willet WC, Manson JE.Physical activity and mortality among women. Am J Public Health 2001; 91:578-583 \\ 4. Bijnem F, Caspersen C. Feskens J. Physical activity and 10-year mortality from cardiovascular disease and all causes. The Zutphen Ederly Study. Arch Intern Med. 1998;159:1499-1505
}

